\title{
Using Hexoskin Wearable Technology to Obtain Body Metrics During Trail Hiking
}

\author{
JEFF MONTES ${ }^{\dagger 1}$, TORI M. STONE ${ }^{\dagger 1}$, JACOB W. MANNING $\ddagger 2$, DAMON \\ MCCUNE ${ }^{\dagger 1}$, DEBRA K. TACAD ${ }^{\dagger 1}$, JOHN C. YOUNG ${ }^{\ddagger 1}$, MARK DEBELISO ${ }^{\ddagger 2}$, and \\ JAMES W. NAVALTA $¥ 1$
}

${ }^{1}$ Department of Kinesiology and Nutrition Sciences, University of Nevada, Las Vegas, Las Vegas, NV, USA; ${ }^{2}$ Department of Physical Education \& Human Performance, Southern Utah University, Cedar City, UT, USA

†Denotes graduate student author, $\neq$ Denotes professional author

\begin{abstract}
International Journal of Exercise Science 8(4): 425-430, 2015. Use of wearable technology to obtain various body metrics appears to be a trending phenomenon. However there is very little literature supporting the notion that these apparatuses can be used for research purposes in the field. The purpose of this study was to utilize Hexoskin wearable technology shirts (HxS) to obtain data in a pilot study using a trail hiking situation. Ten individuals (male, $\mathrm{n}=4$, female $\mathrm{n}=6$ ) volunteered to participate. On the first day, volunteers completed two approximately flat trail hikes at a self-preferred pace with a 15minute rest between trials. On the second day, participants completed a strenuous uphill hike (17.6\% grade) with a 15-minute rest at the summit and then completed the downhill portion. Body metrics provided by the HxS were average heart rate (HR), maximal HR (MHR), total energy expenditure (EE), average respiratory rate (RR), maximal respiratory rate (MRR), total steps (SC), and cadence (CA). Other measurements obtained were systolic and diastolic blood pressure (SBP, DBP), and ratings of perceived exertion (RPE). Data were analyzed using both one-way repeated measures analysis of variance (ANOVA) with significance accepted at $\mathrm{p} \leq 0.05$ and intraclass correlation coefficients (ICC) for each variable. Both were determined using Statistical Package for the Social Sciences software (SPSS). No significant differences for trail type were noted for MHR $(\mathrm{p}=0.38)$, $R R(\mathrm{p}=0.45)$ or MRR $(\mathrm{p}=0.31)$. The uphill trail elicited significantly elevated HR (up=154 $\pm 24 \mathrm{bpm}$, easy $=118 \pm 11 \mathrm{bpm}$, down=129 $\pm 19 \mathrm{bpm} ; \mathrm{p}=0.04$ ) and EE (up=251 $\pm 78 \mathrm{kcal}$, easy $=124 \pm 38 \mathrm{kcal}$, down $=171 \pm 52 \mathrm{kcal} ; \mathrm{p}=0.02)$. Significant ICC were observed for DBP $(r=0.80, p=0.02)$, RR $(\mathrm{r}=0.98, \mathrm{p}=0.01), \mathrm{SC}(\mathrm{r}=0.97, \mathrm{p}=0.01)$ and $\operatorname{RPE}(\mathrm{r}=0.94, \mathrm{p}=0.01)$. Non-significant correlation were noted for uphill RR vs CA ( $r=0.51, p=0.16)$ or RPE vs SBP $(r=0.03, p=0.94)$, HR ( $r=0.60, p=0.12)$, and MHR ( $r=0.70$, $\mathrm{p}=0.051$ ). We utilized HxS to provide physiological data in an applied setting. It should be noted that HR did not register in 5 out of 10 subjects on the easy trail, and 8 of 10 participants during the uphill hike. Additionally, estimated EE appears to be linked to HR intensity. Future investigations taken in an outdoor environment should take these findings into consideration.
\end{abstract}

KEY WORDS: Attire, devices, trek, outdoor activity

\section{INTRODUCTION}

Utilizing wearable technology to obtain body metrics is a trending phenomenon ( 3 , 5). The ease of obtaining individual measures makes wearable technology an attractive option, however, there is very little literature supporting the notion that these apparatuses can be used for field research. 
Hexoskin wearable technology shirt $(\mathrm{HxS})$ is designed to measure several physiological variables including heart rate $(\mathrm{HR})$, respiratory rate (RR), total energy expenditure (EE), and total steps (SC). In a laboratory-based investigation, the validity of this technology was compared with standard laboratory equipment at intensities up to $80 \%$ of the estimated MHR. Minimal variability was reported and consistency was accepted (4).

While there is evidence the HxS may be valid and reliable in a controlled laboratory setting, its application in an outdoor environment is largely unknown. Therefore, the purpose of this study was to utilize HxS technology to obtain data in various trail hiking situations. We used this opportunity as a means to pilot test the Hexoskin for collecting data in a real-life, outdoor setting.

\section{METHODS}

\section{Participants}

Ten individuals (male $\mathrm{n}=4$, female $\mathrm{n}=6$ ) volunteered to participate (age $=24 \pm 10$ years, height $=1663 \mathrm{~cm}$, mass $=65 \pm 18 \mathrm{~kg}$ ). Prior to involvement in the study, participants provided informed consent that was approved by the institutional review board (Southern Utah University protocol \#13-092014).

\section{Protocol}

The protocol was a modification of a previous investigation completed by our research group (2). On the first day, volunteers completed two easy (class I, Yosemite Decimal System (YDS) $1.82 \mathrm{~km}$ (1.13 mile) trail hikes at a self-preferred pace with a 15-minute rest period between trials. Altitude was measured at 5,446 feet above sea level (4400 Heat Stress Tracker, Kestrel, Boothwyn, PA). Body metrics provided by the HxS (Hexoskin Smart Shirt, Montreal, Canada) were HR, MHR, EE, RR, MRR, SC and cadence (CA). The HxS collects data through a data collection device (DCD) that connects by a plug to the shirt itself. Measurements begin when the DCD is attached and stop when disconnected. The HxS DCD was connected when the subject began the easy trail hike and was disconnected when they reached the finish point. Systolic blood pressure $(\mathrm{SBP})$, diastolic blood pressure (DBP) and ratings of perceived exertion (RPE) was also taken. SBP and DBP were measured with an automatic blood pressure device (Omron, BP742, Kyoto, Japan). RPE utilized the Borg scale of 6-20. SPB, DBP, and RPE was taken at the very beginning (directly before $\mathrm{HxS}$ activation) and immediately at the finish for both easy trail hikes (directly after the $\mathrm{HxS}$ was disconnected) (1).

On the second day, participants completed a strenuous (class I, Yosemite Decimal System (YDS)) $1.82 \mathrm{~km}$ (1.13 mile) uphill hike $(17.6 \%$ grade). After a 15 -minutes rest period at the summit, subjects completed the downhill portion. Initial elevation was 5,757 feet above sea level, and rose to 6,443 feet at the summit. HxS, SPB, DBP, and RPE measurements were taken at the beginning and end of both stages of the strenuous trail hike in a similar manner as the easy trail hikes.

\section{Statistical Analysis}

The dependent variables of average HR, Maximal HR, estimated calories, average breathing rate, maximal breathing rate, steps, cadence and RPE were analyzed 
between trail type (initial easy trail, strenuous uphill, strenuous downhill) using one-way repeated measures analysis of variance (ANOVA) (SPSS, ver. 21.0, Chicago, IL, USA) with significance accepted at $\mathrm{p} \leq 0.05$. Intraclass correlation coefficients (ICC) for each of the previously listed dependent variables as well as SBP, $\mathrm{DBP}$, and RPE were determined using the Reliability Analysis: Intraclass Correlation Coefficient option (two-way mixed model, absolute agreement type) in SPSS. ICC's were considered significant at the $p \leq 0.05$ level. Pearson product moment correlation coefficients ( $r$ ) were determined for each trail condition for relationships between RPE and the dependent variables of SBP, HR, and MHR; and between cadence and RR in SPSS using the bivariate correlation option and significance was accepted at $\mathrm{p} \leq 0.05$.

\section{RESULTS}

Preferred hiking speed uphill was significantly slower $\left(4.54 \pm 0.64 \mathrm{~km} \mathrm{~h}^{-1}\right)$ than the easy trail $\left(5.84 \pm 0.45 \mathrm{~km} \mathrm{~h}^{-1}, \mathrm{p}<0.001\right)$ as well as on the downhill portion of the strenuous trail $\left(5.63 \pm 0.71 \mathrm{~km} \mathrm{~h}^{-1}, \mathrm{p}<0.001\right)$. No difference was observed between the hiking pace on the easy trail or the downhill portion of the strenuous trail $(p=0.80)$. Conversely, ratings of perceived exertion were significantly greater during the uphill portion of the strenuous trail (13.7 \pm 2.4$)$ compared to both the easy trail $(9.9 \pm 1.3, \mathrm{p}<0.001)$ and the downhill portion $(10.4 \pm 2.5, p<0.001)$. There was no difference in RPE between the easy trail or the downhill portion of the strenuous trail $(\mathrm{p}=$ $0.40)$.

International Journal of Exercise Science
The uphill trail elicited significantly elevated HR ( $p=0.04$, see figure 1$)$ and $E E$ compared to the other hiking conditions $(p=0.02$, see figure 2$)$. The downhill portion of the strenuous trail produced significantly increased SC compared to the easy trail only ( $p=0.01$, see figure 3 ). No differences were observed for any other condition ( $p>0.05)$. Additionally, downhill CA was significantly greater when compared to the strenuous uphill portion $(p=0.01$, see figure 4 ), but no differences were observed for any other condition $(p>0.05)$. No significant differences for trail type were noted for MHR (up $=168 \pm 22$ beats $\cdot \mathrm{min}^{-1}$, easy = $162 \pm 22$ beats $\cdot \mathrm{min}^{-1}$, down $=147 \pm 20$ beats $\cdot \mathrm{min}^{-1} ; \mathrm{p}=0.38$ ), RR (up $=38 \pm 17$ Breaths $\cdot \mathrm{min}^{-1}$, easy $=34 \pm 7$ Breaths $\cdot \mathrm{min}^{-1}$, down $=39 \pm 14$ Breaths $\cdot \mathrm{min}^{-1} ; \mathrm{p}=0.45$ ) or MRR (up $=54 \pm 17$ Breaths $\cdot \mathrm{min}^{-1}$, easy $=$ $64 \pm 25$ Breaths $\cdot \mathrm{min}^{-1}$, down $=64 \pm 20$ Breaths $\cdot \mathrm{min}^{-1} ; \mathrm{p}=0.31$ ).

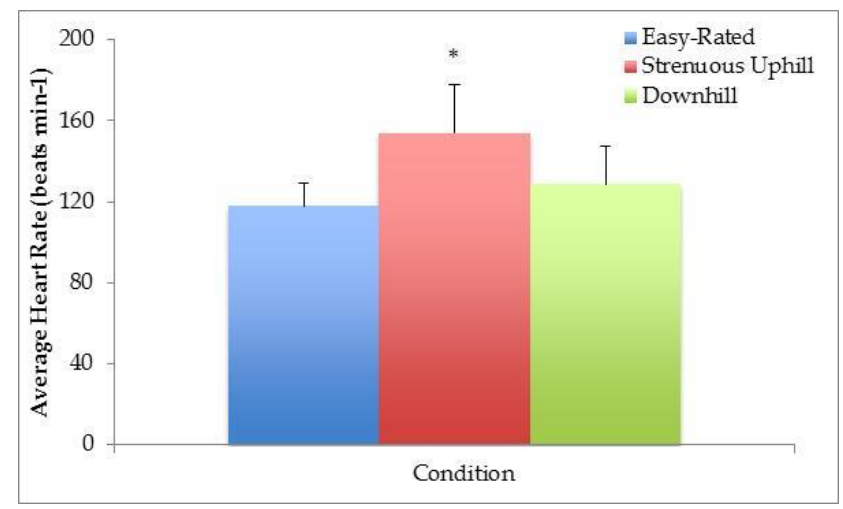

Figure 1. Average heart rate obtained using the Hexoskin shirt on different trail types. * Significantly different from easy-rated and downhill conditions, $\mathrm{P}<0.05$.

Significant ICC was observed for DBP $(\mathrm{r}=$ $0.80, \mathrm{p}=0.02), \mathrm{RR}(\mathrm{r}=0.98, \mathrm{p}=0.01), \mathrm{SC}(\mathrm{r}$ $=0.97, p=0.01), C A(r=0.97, p=0.01)$ and $\operatorname{RPE}(\mathrm{r}=0.94, \mathrm{p}=0.01)$. The ICC for SBP $(\mathrm{r}$ $=0.65, \mathrm{p}=0.07), \mathrm{HR}(\mathrm{r}=0.73, \mathrm{p}=0.14)$, $\operatorname{MHR}(\mathrm{r}=0.65, \mathrm{p}=0.91), \mathrm{EE}(\mathrm{r}=0.53, \mathrm{p}=$ 
$0.25)$, and maximal $R R(r=0.68, p=0.09)$ were not significant.

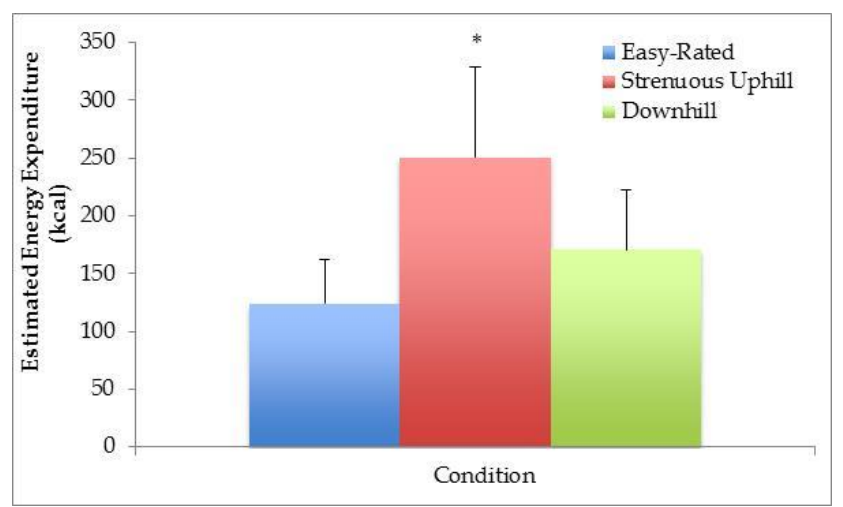

Figure 2. Estimated energy expenditure obtained using the Hexoskin shirt on different trail types. * Significantly different from easy-rated and downhill conditions, $\mathrm{P}<0.05$.

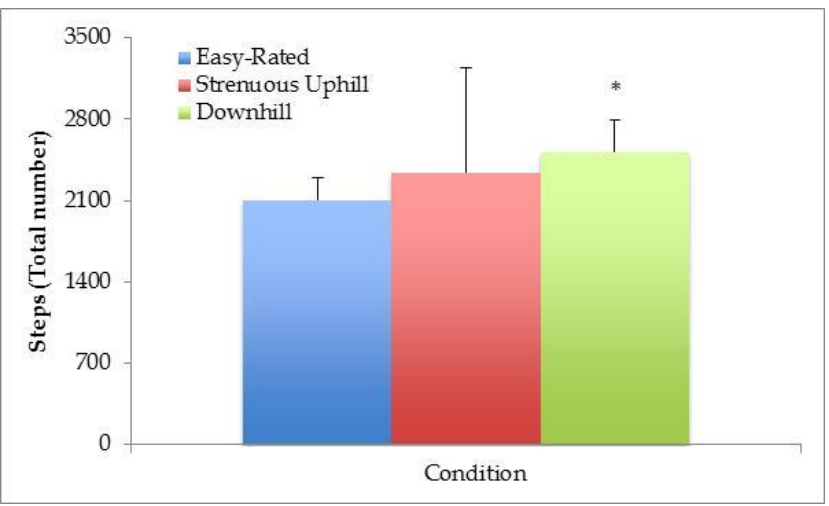

Figure 3. Total step count obtained using the Hexoskin wearable technology on various trails. *Significantly different from the easy-rated trail, $\mathrm{P}<0.05$.

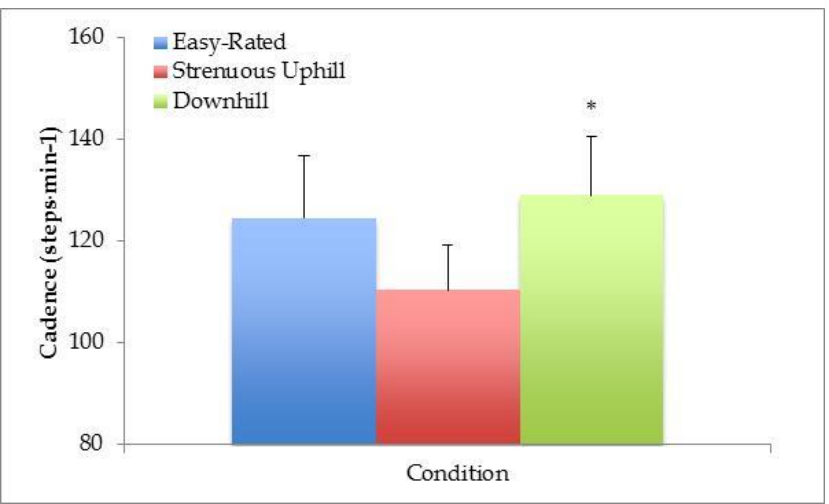

Figure 4. Cadence obtained using the Hexoskin wearable technology on various trails. * Significantly different from the strenuous uphill trail, $\mathrm{P}<0.05$.
Ratings of perceived exertion were not significantly correlated with SBP, average HR, or MHR during any of the hiking stages (see table 1). Furthermore, there was no significant correlation between RR and $\mathrm{CA}$ in any of the hiking stages (easy trail $\mathrm{r}=$ $0.19, \mathrm{p}=0.49$; strenuous uphill $\mathrm{r}=0.52, \mathrm{p}=$ 0.16 ; strenuous downhill $\mathrm{r}=0.25, \mathrm{p}=0.49$ ).

Table 1. Pearson correlations between ratings of perceived exertion and select dependent variable on differently rated trails (easy, strenuous uphill, strenuous downhill).

\begin{tabular}{lccc}
\hline & SBP & $\begin{array}{c}\text { Average } \\
\text { HR }\end{array}$ & $\begin{array}{c}\text { Maximal } \\
\text { HR }\end{array}$ \\
\hline RPE Easy & $\mathrm{r}=0.04$, & $\mathrm{r}=0.29$, & $\mathrm{r}=0.26$, \\
Trail & $\mathrm{p}=0.86$ & $\mathrm{p}=0.37$ & $\mathrm{p}=0.41$ \\
RPE Uphill & $\mathrm{r}=0.03$, & $\mathrm{r}=0.60$, & $\mathrm{r}=0.70$, \\
& $\mathrm{p}=0.94$ & $\mathrm{p}=0.12$ & $\mathrm{p}=0.051$ \\
RPE & $\mathrm{r}=-0.50$, & $\mathrm{r}=0.20$, & $\mathrm{r}=0.30$, \\
Downhill & $\mathrm{p}=0.14$ & $\mathrm{p}=0.61$ & $\mathrm{p}=0.43$ \\
\hline
\end{tabular}

\section{DISCUSSION}

The primary purpose of this investigation was to pilot test the HxS while obtaining physiological measurements in an outdoor trail hiking setting. We hypothesized this technology would allow us to record measures that provided face validity. While measurements of HR and EE demonstrated expected values, it was not the case for MHR, RR, or MRR. Additionally, while HxS measurements of RR, SC, and CA were found to be reliable, the measurements of HR, MHR, EE and MRR returned nonsignificant intraclass correlation coefficients.

Based on the physiological responses that we reported in our previous investigation (2), we expected to observe a general increase during strenuous uphill hiking when compared with both the easy-rated trail and downhill portion of the strenuous 
trail. While we did observe this phenomenon for HR and EE (see figures 1 and 2), it was not consistent for MHR, RR, or MRR. The similar response in these variables to the different trail conditions may be due to the subjects self-selecting a slower pace for the strenuous uphill hike. Evidence for this is suggested by the lower cadence for the uphill hike (Fig. 4) coupled with a significantly greater RPE. Additionally, while not significant, there was a trend for RPE obtained during the uphill strenuous portion of the hike to be correlated with maximal heart rate $(p=0.051)$. We have also observed that the HxS occasionally returned spurious values which could account for the results obtained. This should be taken into account for investigators wishing to utilize $\mathrm{HxS}$ in the field.

While we acknowledge that a great number of subjects are necessary to determine reliability measures for the HxS, the poor ICCs in the current investigation are a concern. This is another factor that should be taken into consideration for researchers using this technology to obtain physiological measures in an outdoor field setting. Future studies similar to work by Villar et al. (4) will be necessary to confirm that the HxS technology is valid and reliable in both laboratory and field-based settings.

The results of this study indicate that HxS technology may be utilized to provide select physiological data in an applied setting. However, our results should interpreted carefully. During the course of our testing, HR did not register in 5 out of 10 subjects on the easy trail, and 8 out of 10 participants during the strenuous hike. Due to the nature of field testing, we were not cognizant of this fact until we attempted to download the data at a later time. Additionally, estimated EE values for the Hexoskin appears to be linked to HR intensity. While further testing is necessary to determine the validity of this algorithm, the returned EE will not be accurate in cases where HR does not register on the $\mathrm{HxS}$ device.

This study demonstrated there may be issues concerning the $\mathrm{HxS}^{\prime} \mathrm{s}$ ability to measure and record data in a real-life setting. This product should first be validated against established laboratory and field standards in order to confirm the manufacturer's claims that the HxS is indeed a useful tool for "physical training, sleep, and personal daily activities." In conclusion, we recommend that validity and reliability be established before $\mathrm{HxS}$ are utilized for research purposes in a fieldbased environment.

\section{REFERENCES}

1. Borg GA. Psychophysical bases of perceived exertion. Med Sci Sports Exerc 14(5):377-381, 1982.

2. Manning JW, Montes J, Stone TM, Rietjens RW, Young JC, DeBeliso M, Navalta JW. Cardiovascular and perceived exertion responses to leisure trail hiking. J Outdoor Rec Ed Leadership. 7(2): 83-92, 2015.

3. Papi E, Osei-Kuffour D, Chen YM, McGregor AH. Use of wearable technology for performance assessment: a validation study. Medical Engineer Physics 37(7):698-704, 2015.

4. Villar R, Beltrame T, Hughson RL. Validation of the Hexoskin wearable vest during lying, sitting, standing and walking activities. Appl Physiol Nutr Metabol In Press, 2015. 
USING HEXOSKIN WEARABLE TECHNOLOGY TO OBTAIN BODY METRICS

5. Yang CC, Hsu YL. A review of accelerometrybased wearable motion detectors for physical activity monitoring. Sensors 10(8):7772-7788, 2010. 